\title{
Existence of positive solutions for second order $m$-point boundary value problems
}

\author{
by RUYun Ma (Lanzhou)
}

\begin{abstract}
Let $\alpha, \beta, \gamma, \delta \geq 0$ and $\varrho:=\gamma \beta+\alpha \gamma+\alpha \delta>0$. Let $\psi(t)=\beta+\alpha t$, $\phi(t)=\gamma+\delta-\gamma t, t \in[0,1]$. We study the existence of positive solutions for the $m$-point boundary value problem$$
\left\{\begin{array}{l}
u^{\prime \prime}+h(t) f(u)=0, \quad 0<t<1, \\
\alpha u(0)-\beta u^{\prime}(0)=\sum_{i=1}^{m-2} a_{i} u\left(\xi_{i}\right), \\
\gamma u(1)+\delta u^{\prime}(1)=\sum_{i=1}^{m-2} b_{i} u\left(\xi_{i}\right),
\end{array}\right.
$$

where $\xi_{i} \in(0,1), a_{i}, b_{i} \in(0, \infty)$ (for $\left.i \in\{1, \ldots, m-2\}\right)$ are given constants satisfying $\varrho-\sum_{i=1}^{m-2} a_{i} \phi\left(\xi_{i}\right)>0, \varrho-\sum_{i=1}^{m-2} b_{i} \psi\left(\xi_{i}\right)>0$ and

$$
\Delta:=\left|\begin{array}{cc}
-\sum_{i=1}^{m-2} a_{i} \psi\left(\xi_{i}\right) & \varrho-\sum_{i=1}^{m-2} a_{i} \phi\left(\xi_{i}\right) \\
\varrho-\sum_{i=1}^{m-2} b_{i} \psi\left(\xi_{i}\right) & -\sum_{i=1}^{m-2} b_{i} \phi\left(\xi_{i}\right)
\end{array}\right|<0 .
$$

We show the existence of positive solutions if $f$ is either superlinear or sublinear by a simple application of a fixed point theorem in cones. Our result extends a result established by Erbe and Wang for two-point BVPs and a result established by the author for three-point BVPs.
\end{abstract}

1. Introduction. The study of multi-point boundary value problems for linear second order ordinary differential equations was initiated by Il'in and Moiseev [6]. Motivated by [6], Gupta [4] studied certain three-point boundary value problems for nonlinear ordinary differential equations. Since then, more general nonlinear multi-point boundary value problems have been studied by several authors. We refer the reader to $[4-6,8-10]$ for some relevant references.

2000 Mathematics Subject Classification: 34B10, 34B18.

Key words and phrases: multi-point boundary value problems, positive solutions, fixed point theorem, cones.

Supported by the NSFC and GG-110-10736-1003 and NWNU-KJCXGC-212. 
In this paper, we are interested in the existence of positive solutions of the second order $m$-point boundary value problem

$$
\left\{\begin{array}{l}
u^{\prime \prime}+h(t) f(u)=0, \quad 0<t<1, \\
\alpha u(0)-\beta u^{\prime}(0)=\sum_{i=1}^{m-2} a_{i} u\left(\xi_{i}\right), \\
\gamma u(1)+\delta u^{\prime}(1)=\sum_{i=1}^{m-2} b_{i} u\left(\xi_{i}\right),
\end{array}\right.
$$

where $\xi_{i} \in(0,1), a_{i}, b_{i} \in(0, \infty)$ (for $\left.i \in\{1, \ldots, m-2\}\right)$ are given constants.

If $a_{i}=b_{i}=0$ for $i=1, \ldots, m-2$, then the $m$-point BVP (1.1) reduces to the two-point BVP

$$
\left\{\begin{array}{l}
u^{\prime \prime}+h(t) f(u)=0, \quad 0<t<1, \\
\alpha u(0)-\beta u^{\prime}(0)=0, \\
\gamma u(1)+\delta u^{\prime}(1)=0 .
\end{array}\right.
$$

In 1994, Erbe and Wang [3] obtained the following excellent result for (1.2).

Theorem A ([3, Theorem 1]). Suppose that

(A1) $f \in C([0, \infty),[0, \infty))$;

(A2) $h \in C([0,1],[0, \infty))$ and $h(t) \equiv 0$ on no subinterval of $(0,1)$;

(A3) $\alpha, \beta, \gamma, \delta \geq 0$, and $\varrho:=\gamma \beta+\alpha \gamma+\alpha \delta>0$.

Then (1.2) has at least one positive solution if either

(i) $f_{0}=0$ and $f_{\infty}=\infty$, or

(ii) $f_{0}=\infty$ and $f_{\infty}=0$,

where

$$
f_{0}:=\lim _{u \rightarrow 0^{+}} \frac{f(u)}{u}, \quad f_{\infty}:=\lim _{u \rightarrow \infty} \frac{f(u)}{u} .
$$

This result has been extended and developed by many authors (see Erbe, $\mathrm{Hu}$ and Wang [2] and Lian, Wong and Yeh [7] for some references).

If $\alpha=\gamma=1, \beta=\delta=0, a_{i}=0$ for $i=1, \ldots, m-2$, and $b_{j}=0$ for $j=2, \ldots, m-2$, then (1.1) reduces to the three-point BVP

$$
\left\{\begin{array}{l}
u^{\prime \prime}+h(t) f(u)=0, \quad 0<t<1, \\
u(0)=0, \quad u(1)=b u(\xi) .
\end{array}\right.
$$

In 1998, Ma [8] obtained the following result for (1.3).

Theorem B ([8, Theorem 1]). Suppose that

(H1) $0<b \xi<1$;

(H2) $f \in C([0, \infty),[0, \infty))$;

(H3) $h \in C([0,1],[0, \infty))$ and there exists $t_{0} \in[\xi, 1]$ such that $h\left(t_{0}\right)>0$. 
Then (1.3) has at least one positive solution if either

(i) $f_{0}=0$ and $f_{\infty}=\infty$, or

(ii) $f_{0}=\infty$ and $f_{\infty}=0$.

Theorem B has been extended by Webb [10]. We remark that in the proof of Theorem B, we rewrite (1.3) as the equivalent integral equation

$$
\begin{aligned}
u(t)= & -\int_{0}^{t}(t-s) h(s) f(u(s)) d s-\frac{b t}{1-b \xi} \int_{0}^{\xi}(\xi-s) h(s) f(u(s)) d s \\
& +\frac{t}{1-b \xi} \int_{0}^{1}(1-s) h(s) f(u(s)) d s
\end{aligned}
$$

which contains one positive term and two negative terms and is not convenient for studying the existence of positive solutions.

In this paper, we consider the more general $m$-point BVP (1.1). To deal with (1.1), we give a new integral equation which is equivalent to (1.1) and only contains two positive terms. Our main result (see Theorem 3.1 below) extends and unifies the main results of $[2,3,7,8]$.

By a positive solution of (1.1) we understand a function $u(t)$ which is positive on $(0,1)$ and satisfies the differential equation and the boundary conditions in (1.1).

The main tool of this paper is the following well-known Guo-Krasnosel'skil fixed point theorem.

Theorem C (see [3]). Let $E$ be a Banach space, and let $K \subset E$ be a cone. Assume $\Omega_{1}, \Omega_{2}$ are open bounded subsets of $E$ with $0 \in \Omega_{1}, \bar{\Omega}_{1} \subset \Omega_{2}$, and let

$$
A: K \cap\left(\bar{\Omega}_{2} \backslash \Omega_{1}\right) \rightarrow K
$$

be a completely continuous operator such that either

(i) $\|A u\| \leq\|u\|, u \in K \cap \partial \Omega_{1}$, and $\|A u\| \geq\|u\|, u \in K \cap \partial \Omega_{2}$; or

(ii) $\|A u\| \geq\|u\|, u \in K \cap \partial \Omega_{1}$, and $\|A u\| \leq\|u\|, u \in K \cap \partial \Omega_{2}$.

Then $A$ has a fixed point in $K \cap\left(\bar{\Omega}_{2} \backslash \Omega_{1}\right)$.

\section{The preliminary lemmas. Set}

$$
\psi(t):=\beta+\alpha t, \quad \phi(t):=\gamma+\delta-\gamma t, \quad t \in[0,1],
$$

and

$$
\Delta:=\left|\begin{array}{cc}
-\sum_{i=1}^{m-2} a_{i} \psi\left(\xi_{i}\right) & \varrho-\sum_{i=1}^{m-2} a_{i} \phi\left(\xi_{i}\right) \\
\varrho-\sum_{i=1}^{m-2} b_{i} \psi\left(\xi_{i}\right) & -\sum_{i=1}^{m-2} b_{i} \phi\left(\xi_{i}\right)
\end{array}\right| .
$$


Lemma 2.1. Let (A3) hold. Assume

(H4)

$$
\Delta \neq 0 \text {. }
$$

Then for $y \in C[0,1]$, the problem

$$
\left\{\begin{array}{l}
u^{\prime \prime}+y(t)=0, \quad 0<t<1 \\
\alpha u(0)-\beta u^{\prime}(0)=\sum_{i=1}^{m-2} a_{i} u\left(\xi_{i}\right), \\
\gamma u(1)+\delta u^{\prime}(1)=\sum_{i=1}^{m-2} b_{i} u\left(\xi_{i}\right)
\end{array}\right.
$$

has a unique solution

$$
u(t)=\int_{0}^{1} G(t, s) y(s) d s+A(y) \psi(t)+B(y) \phi(t)
$$

where

$$
\begin{aligned}
G(t, s) & :=\frac{1}{\varrho} \begin{cases}\phi(t) \psi(s), & 0<s<t<1, \\
\phi(s) \psi(t), & 0<t<s<1,\end{cases} \\
A(y) & :=\frac{1}{\Delta}\left|\begin{array}{ll}
\sum_{i=1}^{m-2} a_{i} \int_{0}^{1} G\left(\xi_{i}, s\right) y(s) d s \quad \varrho-\sum_{i=1}^{m-2} a_{i} \phi\left(\xi_{i}\right) \\
\sum_{i=1}^{m-2} b_{i} \int_{0}^{1} G\left(\xi_{i}, s\right) y(s) d s & -\sum_{i=1}^{m-2} b_{i} \phi\left(\xi_{i}\right)
\end{array}\right| \\
B(y) & :=\frac{1}{\Delta}\left|\begin{array}{ll}
-\sum_{i=1}^{m-2} a_{i} \psi\left(\xi_{i}\right) & \sum_{i=1}^{m-2} a_{i} \int_{0}^{1} G\left(\xi_{i}, s\right) y(s) d s \\
\varrho-\sum_{i=1}^{m-2} b_{i} \psi\left(\xi_{i}\right) & \sum_{i=1}^{m-2} b_{i} \int_{0}^{1} G\left(\xi_{i}, s\right) y(s) d s
\end{array}\right| .
\end{aligned}
$$

Proof. Since $\psi$ and $\phi$ are two linearly independent solutions of the equation $u^{\prime \prime}=0$, we know that any solution of $u^{\prime \prime}(t)=y(t)$ can be represented as

$$
u(t)=\int_{0}^{1} G(t, s) y(s) d s+A \psi(t)+B \phi(t)
$$

where $G$ is as in (2.4).

It is easy to check that the function defined by (2.7) is a solution of (2.2) if $A$ and $B$ are defined by (2.5) and (2.6), respectively.

Now we show that the function defined by (2.7) is a solution of (2.2) only if $A$ and $B$ are as in (2.5) and (2.6), respectively. 
Let $u$ as in (2.7) be a solution of (2.2). Then

$$
\begin{aligned}
u(t)= & \int_{0}^{t} \frac{1}{\varrho} \psi(s) \phi(t) y(s) d s+\int_{t}^{1} \frac{1}{\varrho} \phi(s) \psi(t) y(s) d s+A \psi(t)+B \phi(t), \\
u^{\prime}(t)= & \phi^{\prime}(t) \int_{0}^{t} \frac{1}{\varrho} \psi(s) y(s) d s+\psi^{\prime}(t) \int_{t}^{1} \frac{1}{\varrho} \phi(s) y(s) d s+A \psi^{\prime}(t)+B \phi^{\prime}(t), \\
u^{\prime \prime}(t)= & \phi^{\prime \prime}(t) \int_{0}^{t} \frac{1}{\varrho} \psi(s) y(s) d s+\phi^{\prime}(t) \frac{1}{\varrho} \psi(t) y(t) \\
& +\psi^{\prime \prime}(t) \int_{t}^{1} \frac{1}{\varrho} \phi(s) y(s) d s-\psi^{\prime}(t) \frac{1}{\varrho} \phi(t) y(t)+A \psi^{\prime \prime}(t)+B \phi^{\prime \prime}(t),
\end{aligned}
$$

so that

$$
u^{\prime \prime}(t)=\frac{1}{\varrho}\left[\psi(t) \phi^{\prime}(t)-\phi(t) \psi^{\prime}(t)\right] y(t)=-y(t) .
$$

Since

we have

$$
\begin{aligned}
u(0) & =\beta \int_{0}^{1} \frac{1}{\varrho} \phi(s) y(s) d s+A \beta+B(\gamma+\delta), \\
u^{\prime}(0) & =\alpha \int_{0}^{1} \frac{1}{\varrho} \phi(s) y(s) d s+A \alpha+B(-\gamma),
\end{aligned}
$$

$$
B(\gamma \alpha+\delta \alpha+\gamma \beta)=\sum_{i=1}^{m-2} a_{i}\left[\int_{0}^{1} G\left(\xi_{i}, s\right) y(s) d s+A \psi\left(\xi_{i}\right)+B \phi\left(\xi_{i}\right)\right] .
$$

Since

$$
\begin{array}{r}
u(1)=\delta \int_{0}^{1} \frac{1}{\varrho} \psi(s) y(s) d s+A(\alpha+\beta)+B \delta, \\
u^{\prime}(1)=-\gamma \int_{0}^{1} \frac{1}{\varrho} \psi(s) y(s) d s+A \alpha+B(-\gamma),
\end{array}
$$

we have

$$
A(\gamma \alpha+\delta \alpha+\gamma \beta)=\sum_{i=1}^{m-2} b_{i}\left[\int_{0}^{1} G\left(\xi_{i}, s\right) y(s) d s+A \psi\left(\xi_{i}\right)+B \phi\left(\xi_{i}\right)\right] .
$$

From (2.9) and (2.10), we get

$$
\left\{\begin{array}{l}
{\left[-\sum_{i=1}^{m-2} a_{i} \psi\left(\xi_{i}\right)\right] A+\left[\varrho-\sum_{i=1}^{m-2} a_{i} \phi\left(\xi_{i}\right)\right] B=\sum_{i=1}^{m-2} a_{i} \int_{0}^{1} G\left(\xi_{i}, s\right) y(s) d s} \\
{\left[\varrho-\sum_{i=1}^{m-2} b_{i} \psi\left(\xi_{i}\right)\right] A-\left[\sum_{i=1}^{m-2} b_{i} \phi\left(\xi_{i}\right)\right] B=\sum_{i=1}^{m-2} b_{i} \int_{0}^{1} G\left(\xi_{i}, s\right) y(s) d s}
\end{array}\right.
$$


which implies $A$ and $B$ satisfy (2.5) and (2.6), respectively. This completes the proof of the lemma.

In the following, we will make the following assumption:

$$
\Delta<0, \quad \varrho-\sum_{i=1}^{m-2} a_{i} \phi\left(\xi_{i}\right)>0, \quad \varrho-\sum_{i=1}^{m-2} b_{i} \psi\left(\xi_{i}\right)>0 .
$$

It is easy to see that if $\alpha=\gamma=1, \beta=\delta=0, a_{i}=0$ for $i=1, \ldots, m-2$, $b_{1}>0$ and $b_{j}=0$ for $j=2, \ldots, m-2$, then (H5) reduces to

$$
0<b_{1} \xi_{1}<1
$$

which is a key condition in [8, Theorem 1].

Lemma 2.2. Let (A3) and (H5) hold. Then for $y \in C[0,1]$ with $y \geq 0$, the unique solution $u$ of the problem (2.2) satisfies

$$
u(t) \geq 0, \quad t \in[0,1]
$$

Proof. This is an immediate consequence of the facts that $G \geq 0$ on $[0,1] \times[0,1]$ and $A(y) \geq 0, B(y) \geq 0$.

We note that if (H5) does not hold, then $y \in C[0,1]$ with $y \geq 0$ does not imply that the unique solution $u$ of $(2.2)$ is positive. We can see this from the following result:

LEMma 2.3 ([8, Lemma 3]). Let $b \xi>1$. If $y \in C[0,1]$ and $y \geq 0$, then (1.3) has no positive solution.

LEMma 2.4. Let (A3) and (H5) hold. Let $\sigma \in(0,1 / 2)$ be a constant. Then for $y \in C[0,1]$ with $y \geq 0$, the unique solution $u$ of the problem $(2.2)$ satisfies

$$
\min \{u(t) \mid t \in[\sigma, 1-\sigma]\} \geq \Gamma\|u\|
$$

where $\|u\|=\max \{u(t) \mid t \in[0,1]\}$ and

$$
\Gamma:=\min \{\phi(1-\sigma) / \phi(0), \psi(\sigma) / \psi(1)\} .
$$

Proof. We see from (2.4) and (2.3) that

$$
0 \leq G(t, s) \leq G(s, s), \quad t \in[0,1],
$$

which implies

$$
u(t) \leq \int_{0}^{1} G(s, s) y(s) d s+A(y) \psi(t)+B(y) \phi(t), \quad t \in[0,1] .
$$


Applying (2.4), we find that for $t \in[\sigma, 1-\sigma]$,

$$
\begin{aligned}
\frac{G(t, s)}{G(s, s)} & = \begin{cases}\phi(t) / \phi(s), & 0 \leq s \leq t \leq 1, \\
\psi(t) / \psi(s), & 0 \leq t \leq s \leq 1,\end{cases} \\
& \geq \begin{cases}\phi(1-\sigma) / \phi(0), & 0 \leq s \leq t \leq 1-\sigma, \\
\psi(\sigma) / \psi(1), & \sigma \leq t \leq s \leq 1,\end{cases} \\
& \geq \Gamma,
\end{aligned}
$$

where $\Gamma$ is an in (2.11). Thus for $t \in[\sigma, 1-\sigma]$,

$$
\begin{aligned}
u(t) & =\int_{0}^{1} \frac{G(t, s)}{G(s, s)} G(s, s) y(s) d s+A(y) \psi(t)+B(y) \phi(t) \\
& \geq \Gamma \int_{0}^{1} G(s, s) y(s) d s+A(y) \psi(t)+B(y) \phi(t) \\
& \geq \Gamma\left[\int_{0}^{1} G(s, s) y(s) d s+A(y) \psi(t)+B(y) \phi(t)\right] \geq \Gamma\|u\| .
\end{aligned}
$$

3. The main result. The main result of the paper is the following

Theorem 3.1. Let (H2), (A3) and (H5) hold. Assume that (H6) $h \in C([0,1],[0, \infty))$ and there exists $t_{0} \in[0,1]$ such that $h\left(t_{0}\right)>0$. Then (1.1) has at least one positive solution if either

(i) $f_{0}=0$ and $f_{\infty}=\infty$, or

(ii) $f_{0}=\infty$ and $f_{0}=0$.

Remark 3.2. Condition (H6) is weaker than (H3).

Remark 3.3. Theorem 3.1 extends [3, Theorem 1] and [8, Theorem 1].

Proof of Theorem 3.1. Since $h \in C[0,1]$, we may assume that $t_{0} \in(0,1)$ in (H6). Take $\sigma \in(0,1 / 2)>0$ such that $t_{0} \in(\sigma, 1-\sigma)$ and let $\Gamma$ be defined by $(2.11)$.

Superlinear case. Suppose then that $f_{0}=0$ and $f_{\infty}=\infty$. We wish to show the existence of a positive solution of (1.1). Now (1.1) has a solution $u=u(t)$ if and only if $u$ solves the operator equation

$$
\begin{aligned}
u(t)= & \int_{0}^{1} G(t, s) h(s) f(u(s)) d s+A(h(\cdot) f(u(\cdot))) \psi(t) \\
& +B(h(\cdot) f(u(\cdot))) \phi(t) \\
:= & (T u)(t)
\end{aligned}
$$

where $\phi$ and $\psi, G, A$ and $B$ are defined by (2.1), (2.4), (2.5) and (2.6), 
respectively. Clearly

$$
\begin{aligned}
& |A(h(\cdot) f(u(\cdot)))| \\
& \quad \leq \frac{1}{-\Delta}\left|\begin{array}{ll}
\sum_{i=1}^{m-2} a_{i} \int_{0}^{1} G\left(\xi_{i}, s\right) h(s) d s & \varrho-\sum_{i=1}^{m-2} a_{i} \phi\left(\xi_{i}\right) \\
\sum_{i=1}^{m-2} b_{i} \int_{0}^{1} G\left(\xi_{i}, s\right) h(s) d s & -\sum_{i=1}^{m-2} b_{i} \phi\left(\xi_{i}\right)
\end{array}\right|\|f(u)\| \\
& \quad:=\widetilde{A}\|f(u)\|
\end{aligned}
$$

and

$$
\begin{aligned}
& |B(h(\cdot) f(u(\cdot)))| \\
& \leq \frac{1}{-\Delta}\left|\begin{array}{ll}
-\sum_{i=1}^{m-2} a_{i} \psi\left(\xi_{i}\right) & \sum_{i=1}^{m-2} a_{i} \int_{0}^{1} G\left(\xi_{i}, s\right) h(s) d s \\
\varrho-\sum_{i=1}^{m-2} b_{i} \psi\left(\xi_{i}\right) & \sum_{i=1}^{m-2} b_{i} \int_{0}^{1} G\left(\xi_{i}, s\right) h(s) d s
\end{array}\right|\|f(u)\| \\
& :=\widetilde{B}\|f(u)\| .
\end{aligned}
$$

Define

$$
K=\{u \in C[0,1] \mid u \geq 0, \min \{u(t) \mid t \in[\sigma, 1-\sigma]\} \geq \Gamma\|u\|\} .
$$

It is obvious that $K$ is a cone in $C[0,1]$. Moreover, by Lemmas 2.2 and 2.4, $T K \subset K$. It is also easy to check that $T: K \rightarrow K$ is completely continuous.

Now since $f_{0}=0$, we may choose $H_{1}>0$ so that $f(u) \leq \varepsilon u$ for $0<u$ $<H_{1}$, where $\varepsilon>0$ satisfies

$$
\varepsilon\left(\int_{0}^{1} G(s, s) h(s) d s+\widetilde{A}\|\psi\|+\widetilde{B}\|\phi\|\right) \leq 1 .
$$

Thus, if $u \in K$ and $|u|_{0}=H_{1}$, then from (3.1)-(3.5) and the fact that $G(t, s) \leq G(s, s)$ and $0 \leq \psi(t) \leq \psi(1)$, we have

$$
\begin{aligned}
T u(t)= & \int_{0}^{1} G(t, s) h(s) f(u(s)) d s \\
& +A(h(\cdot) f(u(\cdot))) \psi(t)+B(h(\cdot) f(u(\cdot))) \phi(t) \\
\leq & \left(\int_{0}^{1} G(s, s) h(s) d s+\widetilde{A}\|\psi\|+\widetilde{B}\|\phi\|\right)\|f(u)\| \\
\leq & \varepsilon\left(\int_{0}^{1} G(s, s) h(s) d s+\widetilde{A}\|\psi\|+\widetilde{B}\|\phi\|\right)\|u\| \leq\|u\| .
\end{aligned}
$$


Now if we let

$$
\Omega_{1}=\left\{u \in C[0,1] \mid\|u\|<H_{1}\right\},
$$

then (3.6) shows that $\|T u\| \leq\|u\|$ for $u \in K \cap \partial \Omega_{1}$.

Further, since $f_{\infty}=\infty$, there exists $\widehat{H}_{2}>0$ such that $f(u) \geq \varrho_{0} u$ for $u \geq \widehat{H}_{2}$, where $\varrho_{0}>0$ is chosen so that

$$
\varrho_{0} \gamma \int_{\sigma}^{1-\sigma} G\left(t_{0}, s\right) h(s) d s \geq 1 .
$$

Let $H_{2}=\max \left\{2 H_{1}, \widehat{H}_{2} / \Gamma\right\}$ and $\Omega_{2}=\left\{u \in C[0,1]\|\| u \|<H_{2}\right\}$. Then $u \in K$ and $\|u\|=H_{2}$ implies

$$
\min _{\sigma \leq t \leq 1-\sigma} u(t) \geq \Gamma\|u\| \geq \widehat{H}_{2}
$$

and so

$$
\begin{aligned}
T u\left(t_{0}\right)= & \int_{0}^{1} G\left(t_{0}, s\right) h(s) f(u(s)) d s \\
& +A(h(\cdot) f(u(\cdot))) \psi(t)+B(h(\cdot) f(u(\cdot))) \phi(t) \\
\geq & \int_{0}^{1} G\left(t_{0}, s\right) h(s) f(u(s)) d s \geq \int_{\sigma}^{1-\sigma} G\left(t_{0}, s\right) h(s) \varrho_{0} u(s) d s \\
\geq & \varrho_{0} \Gamma \int_{\sigma}^{1-\sigma} G\left(t_{0}, s\right) h(s) d s\|u\| .
\end{aligned}
$$

Hence, $\|T u\| \geq\|u\|$ for $u \in K \cap \partial \Omega_{2}$. Therefore, by the first part of Theorem C, $T$ has a fixed point $u$ in $K \cap\left(\bar{\Omega}_{2} \backslash \Omega_{1}\right)$ such that $H_{1} \leq\|u\| \leq H_{2}$. This completes the superlinear part of the theorem.

Sublinear case. Suppose next that $f_{0}=\infty$ and $f_{\infty}=0$. We first choose $H_{3}>0$ such that $f(y) \geq M y$ for $0<y<H_{3}$, where

$$
M \Gamma \int_{\sigma}^{1-\sigma} G\left(t_{0}, s\right) h(s) d s \geq 1 .
$$

By using the method to get (3.9), we obtain

$$
\begin{aligned}
T u\left(t_{0}\right) & \geq \int_{0}^{1} G\left(t_{0}, s\right) h(s) f(u(s)) d s \\
& \geq \int_{\sigma}^{1-\sigma} G\left(t_{0}, s\right) h(s) M u(s) d s \geq M \Gamma \int_{\sigma}^{1-\sigma} G\left(t_{0}, s\right) h(s) d s\|u\| .
\end{aligned}
$$


Thus, if we let $\Omega_{3}=\left\{u \in C[0,1] \mid\|u\|<H_{3}\right\}$ then

$$
\|T u\| \geq\|u\|, \quad u \in K \cap \partial \Omega_{3} .
$$

Now, since $f_{\infty}=0$, there exists $\widehat{H}_{4}>0$ so that $f(u) \leq \lambda u$ for $u \geq \widehat{H}_{4}$, where $\lambda>0$ satisfies

$$
\lambda\left(\int_{0}^{1} G(s, s) h(s) d s+\widetilde{A}\|\psi\|+\widetilde{B}\|\phi\|\right) \leq 1 .
$$

We consider two cases:

Case 1. Suppose $f$ is bounded, say $f(y) \leq N$ for all $y \in[0, \infty)$. In this case choose

$$
H_{4}=\max \left\{2 H_{3}, N\left(\int_{0}^{1} G(s, s) h(s) d s+\widetilde{A}\|\psi\|+\widetilde{B}\|\phi\|\right)\right\}
$$

so that for $u \in K$ with $\|u\|=H_{4}$ we have

$$
\begin{aligned}
T u(t) & =\int_{0}^{1} G(t, s) h(s) f(u(s)) d s+A(h(\cdot) f(u(\cdot))) \psi(t)+B(h(\cdot) f(u(\cdot))) \phi(t) \\
& \leq\left(\int_{0}^{1} G(s, s) h(s) d s+\widetilde{A}\|\psi\|+\widetilde{B}\|\phi\|\right) N \leq H_{4}
\end{aligned}
$$

and therefore $\|T u\| \leq\|u\|$.

Case 2. If $f$ is unbounded, then we know from (A1) that there exists $H_{4}>\max \left\{2 H_{3}, \widehat{H}_{4} / \Gamma\right\}$ such that

$$
f(y) \leq f\left(H_{4}\right) \quad \text { for } 0<y \leq H_{4} .
$$

Then for $u \in K$ and $\|u\|=H_{4}$, we have

$$
\begin{aligned}
T u(t) & =\int_{0}^{1} G(t, s) h(s) f(u(s)) d s+A(h(\cdot) f(u(\cdot))) \psi(t)+B(h(\cdot) f(u(\cdot))) \phi(t) \\
& \leq\left(\int_{0}^{1} G(s, s) h(s) d s+\widetilde{A}\|\psi\|+\widetilde{B}\|\psi\|\right)\|f(u)\| \\
& \leq \lambda\left(\int_{0}^{1} G(s, s) h(s) d s+\widetilde{A}\|\psi\|+\widetilde{B}\|\psi\|\right)\|u\| \leq H_{4} .
\end{aligned}
$$

Therefore, in either case we may put

$$
\Omega_{4}=\left\{u \in C[0,1] \mid\|u\|<H_{4}\right\},
$$

and for $u \in K \cap \partial \Omega_{4}$ we have $\|T u\| \leq\|u\|$. By the second part of Theorem C, it follows that BVP (1.1) has a positive solution. Thus, we have completed the proof of Theorem 3.1. 
Remark 3.4. Erbe, Hu and Wang [2] and Lian, Wong and Yeh [7] studied the existence of multiple positive solutions of the two-point boundary value problem

$$
\left\{\begin{array}{l}
u^{\prime \prime}+g(t, u)=0, \quad 0<t<1, \\
\alpha u(0)-\beta u^{\prime}(0)=0 \\
\gamma u(1)+\delta u^{\prime}(1)=0
\end{array}\right.
$$

It is easy to see from the proof of Theorem 3.1 that we can apply Lemmas 2.2 and 2.4 to establish the corresponding multiplicity results under condition (H5) for the $m$-point boundary value problem

$$
\left\{\begin{array}{l}
u^{\prime \prime}+g(t, u)=0, \quad 0<t<1, \\
\alpha u(0)-\beta u^{\prime}(0)=\sum_{i=1}^{m-2} a_{i} u\left(\xi_{i}\right), \\
\gamma u(1)+\delta u^{\prime}(1)=\sum_{i=1}^{m-2} b_{i} u\left(\xi_{i}\right),
\end{array}\right.
$$

and extend the multiplicity results of $[2,7]$ without any difficulties.

\section{References}

[1] H. Dang and K. Schmitt, Existence of positive solutions for semilinear elliptic equations in annular domains, Differential Integral Equations 7 (1994), 747-758.

[2] L. H. Erbe, S. C. Hu and H. Y. Wang, Multiple positive solutions of some two-point boundary value problems, J. Math. Anal. Appl. 203 (1994), 640-648.

[3] L. H. Erbe and H. Y. Wang, On the existence of positive solutions of ordinary differential equations, Proc. Amer. Math. Soc. 120 (1994), 743-748.

[4] C. P. Gupta, Solvability of a three-point nonlinear boundary value problem for a second order ordinary differential equation, J. Math. Anal. Appl. 168 (1992), 540551.

[5] —, A generalized multi-point boundary value problem for second order ordinary differential equations, Appl. Math. Comput. 89 (1998), 133-146.

[6] V. A. Il'in and E. I. Moiseev, Nonlocal boundary value problem of the first kind for a Sturm-Liouville operator in its differential and finite difference aspects, Differ. Equ. 23 (1987), 803-810.

[7] W. C. Lian, F. H. Wong and C. C. Yeh, On the existence of positive solutions of nonlinear second order differential equations, Proc. Amer. Math. Soc. 124 (1996), $1117-1126$.

[8] R. Y. Ma, Positive solutions of nonlinear three-point boundary-value problems, Electron. J. Differential Equations 34 (1999), 1-8.

[9] R. Y. Ma and N. Castaneda, Existence of solutions of nonlinear m-point boundaryvalue problems, J. Math. Anal. Appl. 256 (2001), 556-567. 
[10] J. R. L. Webb, Positive solutions of some three-point boundary value problems via fixed point theory, Nonlinear Anal. 47 (2001), 4319-4332.

Department of Mathematics

Northwest Normal University

Lanzhou 730070, Gansu, People's Republic of China

E-mail: mary@nwnu.edu.cn

Reçu par la Rédaction le 28.2.2002

Révisé le 13.4.2002 